\title{
Outcomes of intracutaneous sutures in comparison with intracutaneous staples in cardiac implantable-electronic device pocket closure
}

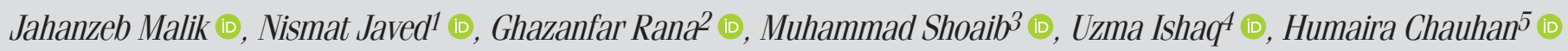 \\ Department of Cardiology, Rawalpindi Institute of Cardiology; Rawalpindi-Pakistan \\ ${ }^{1}$ Department of Medicine, Shifa College of Medicine; Islamabad-Pakistan \\ 2Department of Cardiology, St. Luke's General Hospital; Kilkenny-Ireland \\ ${ }^{3}$ Department of Cardiology, Pakistan Institute of Medical Sciences; Islamabad-Pakistan \\ ${ }^{4}$ Department of Hematology, Foundation University Medical College; Islamabad-Pakistan \\ ${ }^{5}$ Department of Medicine, KRL Hospital Kahuta; Kahuta-Pakistan
}

ABSTRACT

Objective: With the increase in cardiovascular implantable-electronic devices (CIEDs), complications from insertion and healing are also increasing. Therefore, the objective of this study was to compare the intracutaneous stapling method to the absorbable suture technique in terms of complications, procedure time, and pocket closure time.

Methods: An observational study was conducted over the course of three months on patients with CIED implantation. The patients were divided in two groups according to pocket closure technique. Group 1 included patients with pocket closure using intracutaneous sutures; whereas in Group 2, the pocket was closed by intracutaneous staples. Data were collected regarding patient characteristics and wound problems. The endpoints were wound problems, including early and late wound problems (primary), total procedure time, and the time taken for pocket closure (secondary).

Results: One hundred and nineteen patients and 107 patients were allocated to Group 1 and Group 2, respectively. During the three-month observation period, $27(22.6 \%)$ patients in Group 1 and $13(12.1 \%)$ patients in Group 2 suffered from early wound problems, and the combined primary endpoint reached was statistically significant $(p=0.021)$. Minor and major bleeding events were more common in Group 1 [0dds ratio (OR): $4.49, p=0.024 ; 0 R: 0.96, p=0.052$ ]. The time to close the pocket was markedly reduced in Group $2(7.29 \pm 1.42$ vs. $3.98 \pm 1.19, p<0.001)$.

Conclusion: The rate of early wound problems is higher using intracutaneous sutures; and therefore, intracutaneous staples should be preferred to prevent these problems.

Keywords: cardiovascular implantable-electronic devices, pocket pacemaker infection, pacemaker device hematoma, INSORB stapler

Cite this article as: Malik J, Javed N, Rana G, Shoaib M, Ishaq U, Chauhan H. Outcomes of intracutaneous sutures in comparison with intracutaneous staples in cardiac implantable-electronic device pocket closure. Anatol J Cardiol 2021; 25: 716-20.

\section{Introduction}

Over one million cardiovascular implantable-electronic devices (CIED) are being inserted each year worldwide (1). With an increasing number of implantation devices, complications are growing in incidence equally. As the primary focus is on venous access and pacemaker lead related complications, there is paucity of interest on the health of the CIED implantation site. The most common site for CIED pocket is subclavicular cutis where adequate fat tissue for device support and epithelization is available (2). After the procedure, adequate wound care determines the outcome of the functional result as insufficient wound healing opens the door for wound problems like infections or keloid formation. A well-established technique for wound closure is an intracutaneous absorbable suture, which is comfortable for the patients and affects the wound aesthetics minimally, preventing undesirable CIED implantation site complications (3).

Using the same principle of an absorbable suture, a new technique of absorbable staples appears attractive as it can be 


\section{HIGHLIGHTS}

- This is a novel technique to address pocket closure after implantation of cardiac implantable devices using INSORB Stapler which has been used in in breast surgeries and general surgery procedures with better results cosmetically and with respect to local adverse events.

- In our study, we aimed to investigate the early and late problems with INSORB stapler in pocket closure.

- This technique has never been employed on patients with pacemakers. However, it can be an innovation in pocket closure as it is easy and not tedious.

performed quickly and has no needle stick hazard for the operator. Moreover, there is no suture material in the wound that could lead to a potential infectious foci or skin irritation. There are several studies comparing types of sutures with different techniques of wound closure after CIED implant $(4,5)$. However, no prospective data exist in remitting the safety of absorbable intracutaneous staples compared with intracutaneous suture for CIED.

In this study, we aimed to compare outcomes of the standard suture technique for CIED pocket closure with a novel technique of intracutaneous staples.

\section{Methods}

This was an observational study. An Ethical Review Board approval letter was obtained (No: RIC/RERC/12/20), and all the participants signed an informed written consent according to Declaration of Helsinki. Over a period of 1 month, all consecutive patients scheduled for a pacemaker, implantable cardioverterdefibrillator (ICD), or a cardiac resynchronization therapy (CRT) device were prospectively enrolled and followed up for a period of three months for any complications. The patients operated on Monday, Wednesday, and Friday were allocated Group 1, whereas Group 2 included patients operated on Tuesday, Thursday, and Saturday. This was done to match patient numbers and characteristics. There was no operator difference in both the groups. All the procedures were done by an electrophysiologist (JM) at our institute to address the attrition bias. Skin was closed either with an absorbable intracutaneous suture (VICRYLPlus Antibacterial Suture (Ethicon US, LLC.) or with an intracutaneous absorbable stapler (INSORB Skin Stapler, CooperSurgical, Inc. Trumbull, CT). The patients in Group 1 had pocket closure with intracutaneous sutures, and those in Group 2 with staples. The patients were enrolled via consecutive sampling as number of both the techniques for pocket closure was roughly the same in our institute.

All the procedures were performed by our team of electrophysiologists well trained in the use of both the techniques. Hemostasis was secured by diathermy before pocket closure. The devices were secured with a Vicryl suture in both the groups.

Patients with reimplantation of pacing wires or generators, same site reimplantations, complicated diabetes with a history of gangrene, a history of keloid formation, implantation site infections, and subpectoral placement of the generator owing to insufficient subcutis were excluded.

A wound problem log was created perioperatively before discharge and at follow-up appointment after three months. Wound problems were divided into early and late. Insufficient closure, minor/major bleeding, pocket hematoma, and skin irritation leading to encrustation were defined as early wound problems; whereas pocket infection, wound dehiscence, and keloid formation as late wound problems.

The primary endpoint was defined as an early or late wound problem. Early wound problems were defined as complications within the index hospital admission, and late problems were defined as those arising two weeks after the device implantation. In early wound problems, inadequate closure was defined as a disruption of the wound with poor approximation 24 hours after the procedure. Minor bleed was defined as an oozing from the closed wound and major bleed as any bleed not stopped by compression dressing and needing reopening of the wound for assessment and reattachment using an alternative method. Swelling of the pocket post procedure with evidence of collection of blood on ultrasound was defined as pocket hematoma. Encrustation was defined as scaly crusts on the suture site with itching and/or pain. Infection was defined as either a deep tissue infection or a surgical site infection consisting of erythema or discharge from the suture site. Hypertrophy of the surgical wound was considered a keloid. The secondary endpoint was the total procedure time and the time taken to close the pocket after the procedure. Total procedure times were defined as the time from administering a local anesthetic to the application of dressing post procedure. Pocket closure time was defined as the time taken to close the pocket after the deep suture was cut.

\section{Statistical analysis}

Data analysis was performed using the Statistical Package for the Social Sciences version 26 (IBM Corp., Armonk, NY, USA). Continuous variables were presented as mean \pm standard deviation. They were compared using the student's t-test for normal distribution and Mann-Whitney $U$ test for skewed distribution after normality test adjustment with Wilk-Shapiro test. Categorical variables were presented as frequency and percentage. It was compared with the chi-squared and Fischer exact tests when feasible. Relative risk (RR) and confidence interval $(\mathrm{Cl})$ were presented for wound problems. A p value of less than 0.05 was considered significant.

\section{Results}

The study was conducted from February 2020 to April, 2020. One hundred and nineteen patients were allocated Group 1 (51 pacemakers, 44 ICDs, and $16 \mathrm{CRTs}$ ); and 107 patients were 


\begin{tabular}{|c|c|c|c|}
\hline $\begin{array}{l}\text { Baseline } \\
\text { characteristics }\end{array}$ & $\begin{array}{c}\text { Group 1: } \\
\text { Suture }(n=119)\end{array}$ & $\begin{array}{c}\text { Group 2: } \\
\text { Staple }(n=107)\end{array}$ & $P$-value \\
\hline Age (mean \pm SD) & $64.43 \pm 13.19$ & $64.99 \pm 13.35$ & 0.904 \\
\hline Male (\%) & $81(68.1 \%)$ & $70(65.4 \%)$ & 0.673 \\
\hline BMI $($ mean \pm SD) & $27.13 \pm 2.84$ & $27.27 \pm 2.18$ & 0.102 \\
\hline $\begin{array}{l}\text { Coronary artery } \\
\text { disease }\end{array}$ & $62(52.1 \%)$ & $63(58.9 \%)$ & 0.300 \\
\hline $\begin{array}{l}\text { Ischemic } \\
\text { cardiomyopathy }\end{array}$ & $47(39.5 \%)$ & $48(44.9 \%)$ & 0.417 \\
\hline $\begin{array}{l}\text { Dilated } \\
\text { cardiomyopathy }\end{array}$ & $48(40.3 \%)$ & $27(25.2 \%)$ & 0.018 \\
\hline $\begin{array}{l}\text { Hypertensive heart } \\
\text { disease }\end{array}$ & $14(11.8 \%)$ & $20(18.7 \%)$ & 0.143 \\
\hline Diabetes & $70(58.8 \%)$ & $49(41.2 \%)$ & 0.200 \\
\hline Hypertension & $55(46.2 \%)$ & $41(38.3 \%)$ & 0.238 \\
\hline CKD & $36(30.3 \%)$ & $26(24.3 \%)$ & 0.315 \\
\hline Smoking & $45(37.8 \%)$ & $46(43 \%)$ & 0.421 \\
\hline Ejection fraction (\%) & $37.10 \pm 10.70$ & $38.50 \pm 12.17$ & 0.143 \\
\hline Aspirin & $42(35.3 \%)$ & $54(50.5 \%)$ & 0.029 \\
\hline Clopidogrel & $36(30.3 \%)$ & $34(31.8 \%)$ & 0.806 \\
\hline $\begin{array}{l}\text { Aspirin plus } \\
\text { clopidogrel }\end{array}$ & $24(20.2 \%)$ & $33(30.8 \%)$ & 0.061 \\
\hline Warfarin & $13(10.9 \%)$ & $8(7.5 \%)$ & 0.378 \\
\hline Rivaroxaban & $10(8.4 \%)$ & $18(16.8 \%)$ & 0.053 \\
\hline Triple anticoagulation & $3(2.5 \%)$ & $5(4.7 \%)$ & 0.381 \\
\hline
\end{tabular}

\begin{tabular}{|c|c|c|c|}
\hline Devices (\%) & $\begin{array}{c}\text { Group 1: } \\
\text { Suture }(n=119)\end{array}$ & $\begin{array}{c}\text { Group 2: } \\
\text { Staple }(n=107)\end{array}$ & $\boldsymbol{P}$-value \\
\hline \multicolumn{4}{|l|}{ Pacemaker } \\
\hline Single chamber & $9(7.6 \%)$ & $10(9.3 \%)$ & 0.631 \\
\hline Dual chamber & $50(42 \%)$ & $44(41.1 \%)$ & 0.896 \\
\hline ICD & $44(37 \%)$ & $39(36.4 \%)$ & 0.934 \\
\hline CRT & $16(13.4 \%)$ & $14(13.1 \%)$ & 0.938 \\
\hline Procedure time (min) & $62.45 \pm 30.45$ & $58.34 \pm 32.68$ & 0.321 \\
\hline $\begin{array}{l}\text { Pocket closure time } \\
\text { (min) }\end{array}$ & $7.29 \pm 1.42$ & $3.98 \pm 1.19$ & $<0.001$ \\
\hline
\end{tabular}

assigned to Group 2 (54 pacemakers, 39 ICDs, and 14 CRTs). The baseline characteristics are displayed in Table 1, and the procedural details are shown in Table 2.

The patients in both groups were evenly matched for age and sex. More patients in Group 2 were taking aspirin than in Group $1(50.5 \%$ vs. $35.3 \% p=0.029)$. There were no differences regarding underlying heart disease or risk factors for delayed wound

\begin{tabular}{|lccccc|}
\hline Table 3. Early wound problems \\
\hline $\begin{array}{l}\text { Early wound } \\
\text { problems }\end{array}$ & $\begin{array}{c}\text { Group 1: } \\
\text { Suture } \\
(\mathbf{n = 1 1 9})\end{array}$ & $\begin{array}{c}\text { Group 2: } \\
\text { Staple } \\
(\mathbf{n = 1 0 7 )}\end{array}$ & $\mathbf{0 R}$ & $\mathbf{9 5 \%} \mathbf{C l}$ & $\boldsymbol{P}$-value \\
\hline Minor bleed & $10(8.4 \%)$ & $2(1.9 \%)$ & 0.22 & $0.05-0.99$ & 0.024 \\
Major bleed & $4(3.4 \%)$ & 0 & NA & $1.00-1.07$ & 0.052 \\
$\begin{array}{l}\text { Encrustation/ } \\
\text { irritation }\end{array}$ & $3(2.5 \%)$ & $7(6.5 \%)$ & 2.59 & $0.68-9.78$ & 0.147 \\
$\begin{array}{l}\text { Insufficient skin } \\
\text { approximation }\end{array}$ & $1(0.8 \%)$ & $3(2.8 \%)$ & 3.33 & $0.35-31.5$ & 0.266 \\
Hematoma & $9(7.5 \%)$ & $1(0.9 \%)$ & 0.08 & $0.01-0.64$ & 0.013 \\
\hline OR- odds ratio & & & & & \\
\hline
\end{tabular}

Table 4. Late wound problems

\begin{tabular}{|lccccc|}
\hline $\begin{array}{l}\text { Late wound } \\
\text { problems }\end{array}$ & $\begin{array}{c}\text { Group 1: } \\
\text { Suture } \\
(\mathbf{n = 1 1 9 )}\end{array}$ & $\begin{array}{c}\text { Group 2: } \\
\text { Staple } \\
(\mathbf{n = 1 0 7 )}\end{array}$ & $\mathbf{0 R}$ & $\mathbf{9 5 \%} \mathbf{C I}$ & $\boldsymbol{P}$-value \\
\hline Infection & $4(3.4 \%)$ & $2(1.9 \%)$ & 0.55 & $0.10-2.97$ & 0.481 \\
Keloid & $12(10.1 \%)$ & $15(14 \%)$ & 1.39 & $0.68-2.83$ & 0.361 \\
Dehiscence & $5(4.2 \%)$ & $4(3.7 \%)$ & 0.37 & $0.12-1.11$ & 0.634 \\
Skin necrosis & $3(2.5 \%)$ & $3(2.8 \%)$ & 1.11 & $0.22-5.39$ & 0.896 \\
\hline OR - odds ratio & & & & & \\
\hline
\end{tabular}

healing, such as diabetes, chronic kidney disease, or smoking. Moreover, there was no association of bleeding complications with aspirin, clopidogrel, dual antiplatelet therapy (DAPT), or triple anticoagulation. A spurious association of skin necrosis was seen in aspirin users in Group 2 ( $p=0.044)$.

Early endpoints were reached more often with the suture group compared with the staple group ( $26 \%$ vs. $13 \%, p=0.031)$. The early wound problems are differentiated in Table 3 . Minor bleed was seen significantly more in Group 1 than in Group 2 [8.4\% vs. $1.9 \%$, odds ratio (OR) $4.49,95 \% \mathrm{Cl} 0.05-0.99, \mathrm{p}=0.024]$. Only one patient developed a hematoma in Group 2 compared with nine in Group $1(\mathrm{p}=0.013)$. Encrustation or irritation of the wound and insufficient closure were statistically not significant in either group ( $\mathrm{p}=0.147$ and 0.266 respectively). Although major bleeding events were not significant statistically, the staple group did not show any such events, whereas there were four major bleeds in the suture group (OR $0.96,95 \% \mathrm{Cl} 0.93-0.99, \mathrm{p}=0.052$ ).

We did not find any significant difference in late wound problems. However, the incidence of wound dehiscence was more in the suture group $(4.2 \%$ vs. $3.7 \%, 0 \mathrm{R} 2.69,95 \% \mathrm{Cl} 0.89$ $8.11, p$ value $=0.634)$. The late wound problem differentiation is shown in Table 4.

During the three-month observation period, 27 patients $(22.6 \%)$ in Group 1 and 13 patients (12.1\%) in Group 2 suffered from early wound problems, and the combined primary endpoint reached statistical significance $(p=0.021)$. Late wound problems were similar between groups (infection, $\mathrm{p}=0.481$; keloid, $\mathrm{p}=0.361$; dehiscence, $\mathrm{p}=0.634$; skin necrosis, $\mathrm{p}=0.896$ ). We did not find any baseline characteristics to be associated with wound problems. 
The secondary endpoint of total operation time was not significant ( $p=0.321$ ) between the two groups; however, the time it took to close the pocket after device placement was markedly reduced $(7.29 \pm 1.42$ vs. $3.98 \pm 1.19, p<0.001)$ (Table 2$)$.

\section{Discussion}

This prospective observational study was conducted to carry out a head to head comparison of two different modalities of wound closure in CIED. Bleeding and hematoma formation are early complications and can lead to prolonged hospital stay, whereas the most dreaded late complication in these procedures is infection of the pocket. This can affect the integrity of the wound leading to devise explantation, followed by new implantation on the opposite side.

Absorbable staples are an alternative to the conventional subcuticle suture technique. The advantage in minimal handling of the subcutis is increased propensity for accurate hemostasis and less chances of infection. This results in a dry-wound surrounding, which is of extreme importance for a favorable outcome. Moreover, it can be aesthetically agreeable to patients as no suture tracks are present with both the techniques. When performed precisely, the staples can provide adequate wound closure comparable with the tensile strength of multiple sutures.

Regarding early wound problems, we observed insufficient skin approximation after 1 day in $2.8 \%$ of patients in the staple group. This can lead to bacterial nidus from the fluid coming out of the pocket and cause an infection. These patients were then treated with sutures for patient safety, and the dressing was applied for minimal bleeding from the wounds.

Bleeding is a known complication during every procedure that requires an incision. Hypothetically, a suture should impede any bleeding because the tension in the wound makes for a pressure dressing on the pocket. However, superficial stitches are prone to minor or major bleeds leading to hematoma formation in CIED pockets. In this study, we observed a major difference in minor bleeding $(8.4 \%$ vs. $1.9 \%, p=0.02)$ and hematoma formation $(7.5 \%$ vs. $0.9 \%, \mathrm{p}<0.01)$ between the two groups. There was a $20.5 \%$ chance of bleeding in patients taking antiplatelet in one study (6). However, our study suggested no significance between bleeding and antiplatelet therapy.

Wound healing has $f$ distinct phases of hemostasis, inflammation, proliferation, and remodeling. We observed that disruption of skin uniformity by staples disturbs the primary and secondary intention of wound healing and causes excoriation and irritation of the skin causing unfavorable results in the early and late follow-up. However, with sutures, encrustation was rare because of the nondisruptive method.

The study suggests no difference in late wound problems, such as infections, skin necrosis, and keloid formation between the two groups, although dehiscence was more common with intracutaneous sutures. This can be explained by increased tension in the subcutaneous sutures, which causes restricted mobility of the generator and slow fraying of the wound site. It results in the opening of the wound from erosion.
Experiences with INSORB Skin Stapler for wound closure time after CIED is not known as no study has been published so far. Kim et al. (7) prospectively compared 94 patients undergoing breast reconstruction with conventional sutures and INSORB staples. Total operation time was significantly reduced in the staple group $(T=-2.03, p=0.04)$. Our study also demonstrated a significant advantage in terms of the time needed for wound closure with the stapler $(7.29 \pm 1.42$ vs. $3.98 \pm 1.19, p<0.001)$. However, total procedure time was not significantly reduced.

Similar effectiveness in preventing major wound problems has been found in a multicenter prospective study comparing INSORB staples with absorbable thread for dermal suture (8). After a one-year follow-up, the INSORB stapler was found noninferior to the absorbable thread in terms of scar width, suppleness, and hypertrophy. In a randomized clinical trial, the stapler has been reported to be comparable to sutures for caesarian section in terms of wound complications, cosmesis, and total operative times.

The studies mentioned show a substantial benefit and a similar risk profile for the use of the INSORB stapler for wound closure. The data provided in our study shows the superiority of staples compared with suture in the early days after the procedure, which can translate into reduced long-term wound problems.

\section{Study limitations}

The major limitation of this study was the case selection in a non-randomized fashion. Although both groups were equally matched, a randomized clinical trial should have been done for a more accurate assessment of wound problems and cosmetic results in both techniques. The mode of assignment may have had an influence on the outcomes. This can be overcome by proper randomization. Cost-effectiveness was not taken into consideration as there is a marked difference in terms of procedure cost in both the groups. Another limitation was the short observation period of three months. A longer follow-up period would be useful as a deciding factor.

\section{Conclusion}

This study shows a clear benefit in using a skin stapler in comparison to absorbable intracutaneous sutures regarding pocket closure time for CIED implantation. The rate of early wound problems is higher with intracutaneous sutures; however, there was no difference in late wound problems in both the groups.

\section{Conflict of interest: None declared.}

Peer-review: Externally peer-reviewed.

Author contributions: Concept - J.M., N.J., G.R., M.S., U.I., H.C.; Design - J.M., N.J., G.R., M.S., U.I., H.C.; Supervision -M.S.; Fundings J.M., N.J., G.R., M.S., U.I., H.C.; Materials - J.M., N.J., G.R., M.S., U.I., H.C.; Data collection \&/or processing -G.R., U.I.; Analysis \&/or interpretation - J.M., N.J., G.R., M.S., U.I., H.C.; Literature search - J.M., N.J., G.R., M.S., U.I., H.C.; Writing - J.M., N.J., G.R., M.S., U.I., H.C.; Critical review - J.M., N.J., G.R., M.S., U.I., H.C. 


\section{References}

1. Bhatia N, El-Chami M. Leadless pacemakers: a contemporary review. J Geriatr Cardiol 2018; 15: 249-53.

2. Kotsakou M, Kioumis I, Lazaridis G, Pitsiou G, Lampaki S, Papaiwannou A, et al. Pacemaker insertion. Ann Transl Med 2015; 3: 42.

3. Datta G. Pacemaker pocket infection rate and suture technique. Turk Kardiyol Dern Ars 2020; 48: 44-8. [Crossref]

4. De Maria E. New skin closure system facilitates wound healing after cardiovascular implantable electronic device surgery. World J Clin Cases 2015; 3: 675-7. [Crossref]

5. Spencker S, Coban N, Koch L, Schirdewan A, Mueller D. Comparison of skin adhesive and absorbable intracutaneous suture for the implantation of cardiac rhythm devices. Europace 2011; 13: 416-20. [Crossref]
6. Przybylski A, Derejko P, Kwaśniewski W, Urbańczyk-Swić D, Zakrzewska J, Orszulak W, et al. Bleeding complications after pacemaker or cardioverter-defibrillator implantation in patients receiving dual antiplatelet therapy: Results of a prospective, two-centre registry. Neth Heart $\mathrm{J}$ 2010; 18: 230-5. [Crossref]

7. Kim MJ, Park BY, Hong SE, Kang SR. Abstract: Effect of Absorbable Skin Stapler in Autologous Breast Reconstruction Patients: Approach Through Economic and Cosmesis. Plast Reconstr Surg Glob Open 2016; 4 (9 Suppl): 203. [Crossref]

8. Duteille F, Rouif M, Alfandari B, Andreoletti JB, Sinna R, Laurent B, et al. Reduction of skin closure time without loss of healing quality: a multicenter prospective study in 100 patients comparing the use of Insorb absorbable staples with absorbable thread for dermal suture. Surg Innov 2013; 20: 70-3. [Crossref] 